\title{
A Note on the Construction of Metacyclic Extensions
}

\author{
Shin NAKANO and Masahiko SASE \\ Gakushuin University \\ (Communicated by T. Kawasaki)
}

\begin{abstract}
Let $p$ be an odd prime and $r$ a divisor of $p-1$. We present a characterization of metacyclic extensions of degree $p r$ containing a given cyclic extension of degree $r$ over a field of characteristic other than $p$. Furthermore, we give a method of constructing polynomials with Galois groups which are Frobenius groups of degree $p$.
\end{abstract}

\section{Introduction.}

Let $p$ be an odd prime and $r$ a divisor of $p-1$. Let $k$ be a field of characteristic other than $p$. In this note, we investigate metacyclic extensions over $k$ whose Galois groups are given as a semi-direct product $H \ltimes N$, where $H$ and $N$ are cyclic groups of order $r$ and $p$, respectively. We will consider a cyclic extension $K / k$ of degree $r$ satisfying some technical conditions, and classify cyclic extensions over $K$ of degree $p$ which are Galois over $k$, and characterize such metacyclic extensions over $k$ of degree $p r$ in terms of the subextensions of $K(\zeta) / k$, where $\zeta$ is a primitive $p$-th root of unity. The discussion will be done via Kummer extensions over $K(\zeta)$ of degree $p$, for which Cohen's argument in [2, Chapter 5] is useful to us.

The Galois group $G$ of an irreducible polynomial over $k$ of degree $p$ is regarded as a transitive permutation group of degree $p$. Furthermore, as observed by E. Galois himself, such $G$ is a Frobenius group of order $p s$ for some divisor $s$ of $p-1$, provided $G$ is solvable. We shall give a method of generating polynomials of degree $p$ whose Galois groups are Frobenius groups.

This note contains partially the result of Imaoka and Kishi [4]. The authors would like to thank Prof. K. Miyake, Dr. Y. Kishi and Mr. M. Imaoka for their valuable discussions.

\section{The metacyclic group $M_{p}(s \mid r)$.}

Throughout this note, we will fix an odd prime $p$. The field $\mathbf{Z} / p \mathbf{Z}$ of integers modulo $p$ will be denoted $\mathbf{F}_{p}$. Let $r$ be a divisor of $p-1$.

We begin with the definition of a metacyclic group of order $p r$, denoted by $M_{p}(s \mid r)$, as follows. For the details of the group theoretical properties, see for example [3]. Consider a

Received January 23, 2002 
group given by a semi-direct product $H \ltimes N$, where $N$ is a normal subgroup of degree $p$ and $H$ is a cyclic subgroup of degree $r$. This is a metacyclic group with two generators $g$ and $h$ satisfying

$$
g^{p}=h^{r}=1, \quad g h=h g^{x}
$$

where $x$ is regarded as an element of $\mathbf{F}_{p}^{\times}$. In fact, $g, h$ may be taken to be generators of $N$ and $H$, respectively. Let $s$ be the order of $x$. Since $g h^{i}=h^{i} g^{x^{i}}$ for $i \in \mathbf{Z}$, we see that $s$ is a divisor of $r$, and further, the minimum positive integer $i$ such that $h^{i}$ commutes with $g$ is given by $i=s$. It should be noted that the structure of the group is independent of the choice of $x$ and determined by only $r$ and $s$. We denote this group by $M_{p}(s \mid r)$. A Galois extension with Galois group $M_{p}(s \mid r)$ is called an $M_{p}(s \mid r)$-extension.

Let $G$ be a finite group and $N$ a normal subgroup of $G$. Suppose $G / N$ is cyclic and $N$ is abelian. Let $\Gamma_{1}$ and $\Gamma_{2}$ be abelian subgroups of $G$ containing $N$. Then it is easy to show that $\Gamma_{1} \Gamma_{2}$ is also abelian. So there exists the maximum abelian subgroup of $G$ containing $N$.

LEMMA 1. Let $G$ be a finite group and $N$ a normal subgroup of $G$. Assume that $G / N$ and $N$ are cyclic groups of order $r$ and $p$, respectively. Let $s$ be the index of the maximum abelian subgroup of $G$ containing $N$. Then $G=M_{p}(s \mid r)$.

Proof. Let $g$ be a generator of $N$ and take $h \in G$ such that its class in $G / N$ is a generator of $G / N$. Replacing $h$ by its $p$-th power if needed, we have $g^{p}=h^{r}=1$. There is $x \in \mathbf{F}_{p}^{\times}$such that $g h=h g^{x}$. Since $g h^{i}=h^{i} g^{x^{i}}$ for $i \in \mathbf{Z}$, the order of $x$ is given by

$$
\begin{aligned}
& \min \left\{i \mid i>0, x^{i}=1\right\}=\min \left\{i \mid i>0, g h^{i}=h^{i} g\right\} \\
& =\min \{(G: \Gamma) \mid G \supset \Gamma \supset N \text { and } \Gamma \text { is abelian }\} .
\end{aligned}
$$

The last minimum is equal to $s$. Hence we obtain $G=M_{p}(s \mid r)$.

One consequence of this lemma is that $M_{p}(s \mid r)$ and $M_{p}\left(s^{\prime} \mid r\right)$ are never isomorphic if divisors $s, s^{\prime}$ of $r$ are distinct. Besides this, we itemize some properties of $M_{p}(s \mid r)$ as follows:

- $M_{p}(s \mid r)$ is abelian, therefore cyclic, if and only if $s=1$.

- $M_{p}(s \mid r)$ is a Frobenius group if and only if $s=r>1$.

- $M_{p}(2 \mid 2)$ is the dihedral group of order $2 p$.

As mentioned in Introduction, if the Galois group of an irreducible polynomial over $k$ of degree $p$ is solvable, then it is a Frobenius group of order $p s$ for some divisor $s$ of $p-1$. In other words, the Galois group of such a polynomial is $M_{p}(s \mid s)$. We will consider polynomials of this kind, in the last two sections.

\section{Cyclic extensions.}

Let $\zeta$ be a fixed primitive $p$-th root of unity. For a field $F, \tilde{F}$ will mean the $p$-th cyclotomic extension of $F$, that is, $\tilde{F}=F(\zeta)$. For a Galois extension $E / F$, we denote its Galois group by $\operatorname{Gal}(E / F)$. 
Let $K$ be a field of characteristic other than $p$. Put $V(\tilde{K})=\tilde{K}^{\times} / \tilde{K}^{\times p}$ which is considered to be an $\mathbf{F}_{p}$-vector space. Let

$$
\tilde{K}^{\times} \rightarrow V(\tilde{K}), \quad \alpha \mapsto \bar{\alpha}
$$

be the canonical surjective homomorphism. Kummer theory says that any cyclic extension over $\tilde{K}$ of degree $p$ is given by $\tilde{K}(\sqrt[p]{\alpha})$ for some $\alpha \in \tilde{K}^{\times}$. Thus, we have a bijection between the sets of such cyclic extensions and of one-dimensional subspaces of $V(\tilde{K})$. Let $\sigma$ be a generator of $\operatorname{Gal}(\tilde{K} / K)$ and put $d=[\tilde{K}: K]$. We define the injective homomorphism $\chi: \operatorname{Gal}(\tilde{K} / K) \rightarrow \mathbf{F}_{p}^{\times}$by $\zeta^{\sigma}=\zeta^{\chi(\sigma)}$. Let $\varepsilon$ be an idempotent of the group algebra $\mathbf{F}_{p}[\operatorname{Gal}(\tilde{K} / K)]$ defined by

$$
\varepsilon=\frac{1}{d} \sum_{i=0}^{d-1} \chi\left(\sigma^{-i}\right) \sigma^{i}
$$

This is an $\mathbf{F}_{p}$-linear transformation on $V(\tilde{K})$, and its image $V(\tilde{K})^{\varepsilon}$ is the eigenspace of $\sigma$ with the eigenvalue $\chi(\sigma)$, that is,

$$
\bar{\alpha}^{\sigma}=\bar{\alpha}^{\chi(\sigma)} \Leftrightarrow \bar{\alpha} \in V(\tilde{K})^{\varepsilon}
$$

for $\alpha \in \tilde{K}^{\times}$. We define

$$
I(\tilde{K})=\left\{\alpha \in \tilde{K}^{\times} \mid \bar{\alpha} \in V(\tilde{K})^{\varepsilon}\right\} \quad \text { and } \quad I^{*}(\tilde{K})=\left\{\alpha \in I(\tilde{K}) \mid \alpha \notin \tilde{K}^{\times p}\right\} .
$$

The following proposition is known (cf. Cohen [2, Chapter 5]).

PROPOSITION 1. If $L$ is a cyclic extension of degree $p$ over $K$, and $\alpha \in \tilde{K}^{\times}$satisfies $\tilde{L}=\tilde{K}(\sqrt[p]{\alpha})$, then we have $\alpha \in I^{*}(\tilde{K})$. Conversely, for any $\alpha \in I^{*}(\tilde{K}), \tilde{K}(\sqrt[p]{\alpha})$ is an abelian extension over $K$ of degree $d p$ which contains a unique cyclic extension $L$ over $K$ of degree $p$.

Thus there is a bijection between the sets of cyclic extensions over $K$ of degree $p$ and of one-dimensional subspaces of $V(\tilde{K})^{\varepsilon}$.

\section{4. $M_{p}(s \mid r)$-extensions.}

In this section, we consider the case that $K$ has a subfield $k$ such that $K / k$ is a cyclic extension of degree $r$. Let us assume $K / k$ has the following properties:

(A) $K \cap \tilde{k}=k$,

(B) $r>1$ and $r$ is a divisor of $d=[\tilde{K}: K]$.

We will fix such an extension $K / k$ in the following discussion. Under these assumptions, we will characterize the cyclic extensions over $K$ of degree $p$ which are Galois extensions over $k$ with the Galois group $M_{p}(s \mid r)$, that is, $M_{p}(s \mid r)$-extensions over $k$ containing $K$. The degree $[\tilde{k}: k]$ is equal to $d=[\tilde{K}: K]$ by (A). So the four fields $k, K, \tilde{K}$ and $\tilde{k}$ form a "parallelogram". It follows that $\tilde{K} / k$ is abelian and its Galois group is the direct product of those of $\tilde{K} / K$ and $\tilde{K} / \tilde{k}$. Since $d$ divides $p-1$, the assumption (B) implies that the degree $[\tilde{K}: k]=r d$ is prime to $p$. 
We put $V(E)=E^{\times} / E^{\times p}$ also for a subextension $E$ of $\tilde{K} / k$. Since $E^{\times} \cap \tilde{K}^{\times p}=E^{\times p}$, we can regard $V(E)$ as a subspace of $V(\tilde{K})$. Moreover $\operatorname{Gal}(\tilde{K} / k)$ acts on $V(E)$ naturally, so $V(E)$ is an $\mathbf{F}_{p}[\operatorname{Gal}(\tilde{K} / k)]$-module.

LEMMA 2. Let $H$ be a subgroup of $\operatorname{Gal}(\tilde{K} / k)$ and $E$ the subextension of $\tilde{K} / k$ corresponding to $H$. Then, for $\alpha \in \tilde{K}^{\times}$the following properties (i), (ii) are equivalent:

(i) $\bar{\alpha} \in V(E)$.

(ii) $\bar{\alpha} \xi=\bar{\alpha}$ for every $\xi \in H$.

Proof. It is easy to see that (i) implies (ii). Conversely, if $\alpha$ satisfies (ii), then $\bar{\alpha}^{[\tilde{K}: E]}=$ $\overline{N_{\tilde{K} / E}(\alpha)} \in V(E)$. Since $[\tilde{K}: E]$ is prime to $p$, we have $\bar{\alpha} \in V(E)$.

Let $\sigma$ and $\varepsilon$ be as in the previous section. For a subextension $E$ of $\tilde{K} / k$, we also define

$$
I(E)=\left\{\alpha \in \tilde{K}^{\times} \mid \bar{\alpha} \in V(E)^{\varepsilon}\right\} \quad \text { and } \quad I^{*}(E)=\left\{\alpha \in I(E) \mid \alpha \notin \tilde{K}^{\times p}\right\} .
$$

Note that $V(E) \cap V(\tilde{K})^{\varepsilon}=V(E)^{\varepsilon}$ holds, since $\varepsilon$ is an idempotent. Let $\tau$ be a generator of $\operatorname{Gal}(\tilde{K} / \tilde{k})$. Then the Galois group of $\tilde{K} / k$ is generated by $\sigma$ and $\tau$. Let $s$ be a divisor of $r$ and put

For $j \in J_{s}$, we define an element of $\operatorname{Gal}(\tilde{K} / k)$ as

$$
J_{s}=\{j \mid 1 \leq j \leq s,(j, s)=1\} .
$$

$$
\rho(s, j)=\sigma^{d j / s} \tau
$$

and denote by $E(s, j)$ the subextension of $\tilde{K} / k$ corresponding to the cyclic subgroup generated by $\rho(s, j)$.

The main theorem of this note is the following

THEOREM 1. Let L be a cyclic extension of degree $p$ over $K$ and take $\alpha \in I^{*}(\tilde{K})$ with $\tilde{L}=\tilde{K}(\sqrt[p]{\alpha})$.

(1) If $L / k$ is Galois, then $L / k$ is an $M_{p}(s \mid r)$-extension for some divisor $s$ of $r$.

(2) Let $s$ be a divisor of $r$. Then $L / k$ is an $M_{p}(s \mid r)$-extension if and only if $\alpha \in$ $I^{*}(E(s, j))$ for some $j \in J_{s}$.

Since (1) is an immediate consequence of Lemma 1, we shall show (2) only. We need the following two lemmas.

LEMMA 3. Let $F$ be a subfield of $\tilde{K}$ such that $\tilde{K} / F$ is a Galois extension. Then, for $\alpha \in \tilde{K}^{\times}$, the following (i), (ii) are equivalent:

(i) $\tilde{K}(\sqrt[p]{\alpha}) / F$ is a Galois extension.

(ii) For every $\xi \in \operatorname{Gal}(\tilde{K} / F)$, there exists $x \in \mathbf{F}_{p}^{\times}$such that $\bar{\alpha}^{\xi}=\bar{\alpha}^{x}$.

Proof. If $\tilde{K}(\sqrt[p]{\alpha}) / F$ is a Galois extension, then $\tilde{K}\left(\sqrt[p]{\alpha^{\xi}}\right)=\tilde{K}(\sqrt[p]{\alpha})$ for any $\xi \in$ $\operatorname{Gal}(\tilde{K} / F)$. Therefore, from Kummer theory, we see that there exists $x \in \mathbf{F}_{p}^{\times}$such that $\bar{\alpha}^{\xi}=\bar{\alpha}^{x}$. The converse is obvious.

Lemma 4. Suppose $\alpha \in \tilde{K}^{\times}$satisfies $\bar{\alpha}^{\tau}=\bar{\alpha}^{x}$ for some $x \in \mathbf{F}_{p}^{\times}$. If the order of $x$ is equal to $s$, then $\tilde{K}(\sqrt[p]{\alpha}) / \tilde{k}$ is an $M_{p}(s \mid r)$-extension. 
Proof. First we recall that $s$ divides $r=[\tilde{K}: \tilde{k}]$. Let $i$ be a divisor of $r$ and $F_{i}$ the subextension of $\tilde{K} / \tilde{k}$ corresponding to $\left\langle\tau^{i}\right\rangle$. Suppose $x^{i}=1$. Then $\bar{\alpha}^{\tau^{i}}=\bar{\alpha}^{x^{i}}=\bar{\alpha}$, thus $\bar{\alpha} \in V\left(F_{i}\right)$ from Lemma 2. So, there exists $\beta \in F_{i}^{\times}$such that $\bar{\beta}=\bar{\alpha}$, and $\tilde{K}(\sqrt[p]{\alpha})$ contains the cyclic extension $F_{i}(\sqrt[p]{\beta})$ over $F_{i}$ of degree $p$. Hence $\tilde{K}(\sqrt[p]{\alpha}) / F_{i}$ is abelian. Furthermore, it is not difficult to verify the converse. So, $\tilde{K}(\sqrt[p]{\alpha}) / F_{i}$ is abelian if and only if $x^{i}=1$. Therefore $F_{s}$ is the smallest subextension of $\tilde{K} / \tilde{k}$ over which $\tilde{K}(\sqrt[p]{\alpha})$ is abelian. Using Lemma 1, we conclude that $\tilde{K}(\sqrt[p]{\alpha}) / \tilde{k}$ is an $M_{p}(s \mid r)$-extension.

Proof OF THEOREM 1 (2). Assume that $L$ is an $M_{p}(s \mid r)$-extension of $k$. Then $\tilde{L} / \tilde{k}$ is also an $M_{p}(s \mid r)$-extension. Therefore, it follows from Lemmas 3 and 4 that there exists $x \in \mathbf{F}_{p}^{\times}$of order $s$ with $\bar{\alpha}^{\tau}=\bar{\alpha}^{x}$. Since $\chi\left(\sigma^{d / s}\right)$ is of order $s$ as well, we can choose $j \in J_{s}$ satisfying $x \chi\left(\sigma^{d / s}\right)^{j}=1$. Then $\bar{\alpha}^{\rho(s, j)}=\bar{\alpha}^{\sigma^{d j / s} \tau}=\bar{\alpha}^{x \chi\left(\sigma^{d j / s}\right)}=\bar{\alpha}$, and thus $\bar{\alpha} \in V(E(s, j))$ from Lemma 2. So we have $\bar{\alpha} \in V(E(s, j)) \cap V(\tilde{K})^{\varepsilon}=V(E(s, j))^{\varepsilon}$. Hence $\alpha \in I^{*}(E(s, j))$.

Conversely, suppose $\alpha \in I^{*}(E(s, j))$ for some $j \in J_{s}$. Then we have $\bar{\alpha}^{\rho(s, j)}=\bar{\alpha}$. On the other hand, we know the relation $\bar{\alpha}^{\sigma}=\bar{\alpha}^{\chi(\sigma)}$ and the fact that $\operatorname{Gal}(\tilde{K} / k)$ is generated by $\sigma$ and $\rho(s, j)$. Thus, by Lemma 3 , we see that $\tilde{L} / k$ is Galois. So, if $L^{\prime}$ is a conjugate field of $L$ over $k$, then $L^{\prime}$ is contained in $\tilde{L}$ and $\left[L^{\prime}: K\right]=p$, and thus $L^{\prime}$ must coincide with $L$. This means that $L / k$ is Galois. The Galois group of $L / k$ is isomorphic to $\operatorname{Gal}(\tilde{L} / \tilde{k})$. Now we have $\bar{\alpha}^{\tau}=\bar{\alpha}^{\sigma^{-d j / s} \rho(s, j)}=\bar{\alpha}^{\chi\left(\sigma^{-d j / s}\right)}$. Since $j$ is prime to $s$, the order of $\chi\left(\sigma^{-d j / s}\right)$ is equal to $s$. Therefore, by Lemma $4, \tilde{L} / \tilde{k}$ is an $M_{p}(s \mid r)$-extension, and so is $L / k$.

In case $s=1$, the theorem claims that $L / k$ is abelian extension if and only if $\alpha \in I^{*}(\tilde{k})$. The case $r=s=2$ where the Galois groups are dihedral was treated also by Imaoka and Kishi [4].

\section{Defining polynomials for $M_{p}(s \mid r)$-extensions.}

Let notations and assumptions be as in the previous section. We will fix $e \in \mathbf{Z}[G]$ satisfying $y \varepsilon \equiv e \bmod p$ for some $y \in \mathbf{F}_{p}^{\times}$. Then we have

$$
I(E)=\left\{\beta^{e} \gamma^{p} \mid \beta \in E^{\times}, \gamma \in \tilde{K}^{\times}\right\},
$$

for a subextension $E$ of $\tilde{K} / k$.

Now it follows from Proposition 1 that a cyclic extension $L$ over $K$ of degree $p$ is given by $L=K\left(\operatorname{Tr}_{\tilde{L} / L}\left(\sqrt[p]{\beta^{e}}\right)\right)$ with $\beta \in \tilde{K}^{\times}$satisfying $\beta^{e} \notin \tilde{K}^{\times p}$, namely, $\beta^{e} \in I^{*}(\tilde{K})$. For such $\beta$, denote by $f_{\beta}(X)$ the monic minimal polynomial of $\operatorname{Tr}_{\tilde{L} / L}\left(\sqrt[p]{\beta^{e}}\right)$ over $K$. The next lemma on the coefficients of $f_{\beta}(X)$ is obtained by thorough calculations in Cohen [2, Chapter 5].

LEMmA 5. Every coefficient of $f_{\beta}(X)$ of degree less than $p$ is given in the form of a finite sum

$$
\sum_{\nu} c_{\nu} \beta^{z_{v}}, \quad c_{\nu} \in \mathbf{F}_{K}, z_{v} \in \mathbf{Z}[\operatorname{Gal}(\tilde{K} / K)],
$$

where $\mathbf{F}_{K}$ is the prime field contained in $K$. 
Suppose $\beta \in E(s, j)^{\times}$satisfies $\beta^{e} \notin E(s, j)^{\times p}$, where $s$ is a divisor of $r$ and $j \in J_{s}$. Then $\beta^{e} \in I^{*}(E(s, j))$ and, by Theorem 1 , the cyclic extension obtained by adjoining a root of $f_{\beta}(X)$ to $K$ is an $M_{p}(s \mid r)$-extension over $k$. Furthermore, an $M_{p}(s \mid r)$-extension of this kind is always constructed in this manner. Now Lemma 5 implies that $f_{\beta}(X) \in k[X]$, since $K \cap E(s, j)=k$. So we are interested in the minimal splitting field of $f_{\beta}(X)$ over $k$. The Galois group of $f_{\beta}(X)$ needs to be a Frobenius group, that is, $M_{p}(t \mid t)$ with a divisor $t$ of $p-1$. In fact, the following result is obtained in the case $s=r$.

THEOREM 2. Let $j \in J_{r}$ and $\beta \in E(r, j)^{\times}$satisfying $\beta^{e} \notin E(r, j)^{\times p}$. Then $f_{\beta}(X) \in$ $k[X]$ and its minimal splitting field over $k$ is the $M_{p}(r \mid r)$-extension $L$ over $k$ such that $K \subset$ $L \subset \tilde{K}\left(\sqrt[p]{\beta^{e}}\right)$.

PROOF. Let $L_{\beta}$ be the minimal splitting field of $f_{\beta}(X)$ over $k$, and put $K_{\beta}=L_{\beta} \cap K$. Then, since $L_{\beta} / K_{\beta}$ is a cyclic extension of degree $p$, it follows that $L=L_{\beta} K$ is abelian over $K_{\beta}$. However, by Lemma 1 , the $M_{p}(r \mid r)$-extension $L / k$ never contains a subextension $F$ such that $F \subsetneq K$ and $L / F$ is abelian. Thus $K_{\beta}$ must be equal to $K$. Hence we conclude $L_{\beta}=L$.

As for a divisor $s$ of $r$, we have the following

THEOREM 3. Let $s$ be a divisor of $r$ and $j \in J_{s}$. Take $\beta \in E(s, j)^{\times}$such that $\beta^{e} \notin E(s, j)^{\times p}$. Then $f_{\beta}(X) \in k[X]$ and its Galois group over $k$ is isomorphic to $M_{p}(s \mid s)$.

Proof. Let $K_{s}$ be the cyclic extension over $k$ of degree $s$ contained in $K$. Then $\tilde{K}_{s}$ is the subextension of $\tilde{K} / \tilde{k}$ corresponding to the subgroup $\left\langle\tau^{s}\right\rangle$. Since $\tau^{s}=\rho(s, j)^{s} \in$ $\langle\rho(s, j)\rangle$, we have $E(s, j) \subseteq \tilde{K}_{s}$. So, applying the above discussion to the extension $K_{s} / k$ instead of $K / k$, we completes the proof.

Polynomials with Frobenius groups of degree $p$ as Galois groups are studied from another viewpoint, by Bruen, Jensen and Yui [1].

\section{Examples.}

We will illustrate the above results with some numerical examples. Take $k=\mathbf{Q}$ and $p=5$. In this case, $\tilde{\mathbf{Q}}=\mathbf{Q}(\zeta)$ is cyclic over $\mathbf{Q}$ of degree 4. Let $K=\mathbf{Q}(\sqrt{2+\sqrt{2}})$. Then $K / \mathbf{Q}$ is a cyclic extension of degree 4 satisfying the properties $K \cap \tilde{\mathbf{Q}}=\mathbf{Q}$ and $[\tilde{K}: K]=4$. Put

$$
\theta_{1}=\sqrt{2+\sqrt{2}}, \quad \theta_{2}=\sqrt{2-\sqrt{2}}, \quad \theta_{3}=-\sqrt{2-\sqrt{2}}, \quad \theta_{4}=-\sqrt{2+\sqrt{2}} .
$$

We can take generators $\sigma, \tau$ of $\operatorname{Gal}(\tilde{K} / K)$ and $\operatorname{Gal}(\tilde{K} / \tilde{k})$, respectively, such as $\zeta^{\sigma}=\zeta^{2}$ and $\theta_{1}^{\tau}=\theta_{2}$. Then it is easy to check $\theta_{2}^{\tau}=\theta_{4}$ and $\theta_{4}^{\tau}=\theta_{3}$. Now we put $e=3+4 \sigma+2 \sigma^{2}+\sigma^{3}$ which satisfies the congruence $2 \varepsilon \equiv e \bmod 5$. For $\beta \in \tilde{K}^{\times}$satisfying $\beta^{e} \in I^{*}(\tilde{K})$, the minimal polynomial $f_{\beta}(X)$ of $\operatorname{Tr}_{\tilde{L} / L}\left(\sqrt[5]{\beta^{e}}\right)$ is written in the form 


$$
\begin{aligned}
f_{\beta}(X)= & X^{5}-10 N(\beta) X^{3}-5 N(\beta) T\left(\beta^{1+\sigma}\right) X^{2} \\
& +5 N(\beta)\left(N(\beta)-T\left(\beta^{1+2 \sigma+\sigma^{2}}\right)\right) X-N(\beta) T\left(\beta^{2+3 \sigma+\sigma^{2}}\right)
\end{aligned}
$$

with $N=N_{\tilde{K} / K}$ and $T=T r_{\tilde{K} / K}$, which had appeared in Cohen [2, Chapter 5]. Using this, we present several defining polynomials for Frobenius extensions over $\mathbf{Q}$ via $E(4,1), E(4,3)$ and $E(2,1)$.

(1) $\quad E(4,1)=\mathbf{Q}(\xi)$ with $\xi=\theta_{1} \zeta+\theta_{2} \zeta^{2}+\theta_{4} \zeta^{4}+\theta_{3} \zeta^{3}$. If we choose $\beta_{1}=\xi+1$, then $\beta_{1}^{e} \in I^{*}(E(4,1))$ and

$$
f_{\beta_{1}}(X)=X^{5}-310 X^{3}-620 X^{2}+10385 X+20956 .
$$

The Galois group of $f_{\beta_{1}}(X)$ over $\mathbf{Q}$ is $E_{5}(4 \mid 4)$, that is, the Frobenius group of order 20.

(2) $E(4,3)=\mathbf{Q}(\eta)$ with $\eta=\theta_{1} \zeta+\theta_{2} \zeta^{3}+\theta_{4} \zeta^{4}+\theta_{3} \zeta^{2}$. Taking $\beta_{2}=\eta+1$, we have $\beta_{2}^{e} \in I^{*}(E(4,3))$ and

$$
f_{\beta_{2}}(X)=X^{5}-1110 X^{3}-2220 X^{2}+259185 X+75036,
$$

which Galois group over $\mathbf{Q}$ is also the Frobenius group of order 20 .

(3) $E(2,1)=\mathbf{Q}(\omega)$ with $\omega=\sqrt{-5+2 \sqrt{5}} \sqrt{2}$. Put $\beta_{3}=\omega+1$. Then $\beta_{3}^{e} \in$ $I^{*}(E(2,1))$ and

$$
f_{\beta_{3}}(X)=X^{5}-410 X^{3}-820 X^{2}+23985 X-13284 .
$$

The Galois group of $f_{\beta_{3}}(X)$ over $\mathbf{Q}$ is the dihedral group of order 10 .

\section{References}

[ 1 ] A. A. Bruen, C. U. Jensen and N. Yui, Polynomials with Frobenius groups of prime degree as Galois groups II, J. Number Theory 24 (1986), 305-359.

[ 2 ] H. CoHEN, Advanced topics in computational number theory, Springer (2000).

[ 3 ] B. HUPPERT, Endliche Gruppen I, Springer (1967).

[ 4 ] M. IMAOKA and Y. KISHI, Spiegelung relation between dihedral extensions and Frobenius extensions, preprint.

Present Address:

DEPARTMENT OF MATHEMATICS, GAKUSHUIN UNIVERSity,

MEJIRO, TOKYO, 171-8588, JAPAN.

e-mail:shin@math.gakushuin.ac.jp

sasem@math.gakushuin.ac.jp 